\title{
Scaling solutions in the derivative expansion
}

\author{
N. Defenu ${ }^{1, *}$ and A. Codello ${ }^{2,3, \uparrow}$ \\ ${ }^{1}$ Institut für Theoretische Physik, Universität Heidelberg, D-69120 Heidelberg, Germany \\ ${ }^{2} \mathrm{CP}^{3}$-Origins, University of Southern Denmark, Campusvej 55, 5230 Odense M, Denmark \\ ${ }^{3}$ INFN-Sezione di Bologna, via Irnerio 46, 40126 Bologna, Italy
}

(Received 24 February 2018; published 23 July 2018)

\begin{abstract}
Scalar field theories with $\mathbb{Z}_{2}$ symmetry are the traditional playground of critical phenomena. In this work, these models are studied using functional renormalization group (FRG) equations at order $\partial^{2}$ of the derivative expansion and, differently from previous approaches, the spike plot technique is employed to find the relative scaling solutions in two and three dimensions. The anomalous dimension of the first few universality classes in $d=2$ is given, and the phase structure predicted by conformal field theory is recovered (without the imposition of conformal invariance), while in $d=3$ a refined view of the standard Wilson-Fisher fixed point is found. Our study enlightens the strength of shooting techniques in studying FRG equations, suggesting them as candidates to investigate strongly nonperturbative theories even in more complex cases.
\end{abstract}

DOI: $10.1103 /$ PhysRevD.98.016013

\section{INTRODUCTION}

Since the discovery of the phenomenon of universality and its explanation in terms of the renormalization group (RG) [1], one of the main goals of statistical and quantum field theory has been the classification of all universality classes, i.e., the determination of fixed points of the RG flow. Even though the phase diagram of scalar field theories has been subject to intense investigations over the decades, we still lack a complete picture of theory space even in the simplest cases, such as that of single-component scalar theories in two or three dimensions. This is not a surprise since the problem is inherently nonperturbative, but what is also missing is an easy way to render and visualize the complex landscape of theory space, since this is generally an infinite functional space.

In recent years, the functional renormalization group (FRG) $[2,3]$ has shown its versatility and strength as a nonperturbative RG technique in many applications [4-6]. In this approach, the traditional RG is extended to work in the functional space of the effective average action (EAA), i.e., the scale-dependent generator of the one-particle irreducible vertexes of the theory, allowing us to pursue

\footnotetext{
*defenu@thphys.uni-heidelberg.de codello@cp3-origins.net
}

Published by the American Physical Society under the terms of the Creative Commons Attribution 4.0 International license. Further distribution of this work must maintain attribution to the author(s) and the published article's title, journal citation, and DOI. Funded by SCOAP ${ }^{3}$. a new kind of nonperturbative expansion and to overcome the traditional limitations of diagrammatic techniques.

In this paper, we use the flow equations for the EAA at order $O\left(\partial^{2}\right)$ of the derivative expansion to investigate scaling solutions (i.e., functional RG fixed points) of single-component scalar theories with $\mathbb{Z}_{2}$ symmetry in two and three dimensions. Our approach generalizes the spike plot technique already employed to solve the local potential approximation (LPA) in [7-10] and refines the $O\left(\partial^{2}\right)$ study [11] where a power like regulator and numerical relaxation methods were employed. For related studies, but from a Polchinski equation perspective, see [12] and reference therein; for a proper time RG study see [13].

Our technique provides an extension of the shooting method previously employed in the literature to describe single isolate fixed points in $d>2$ [14] to the investigation of the whole phase diagram of the $d=2$ case, where infinitely many fixed points exist. Our analysis paves the way for the systematic application of shooting techniques to high-order truncation in derivative expansions for more complex field theories. In particular, we foresee possible application of the present technique to $O(N)$ symmetric field theories, where strongly nonperturbative fixed point exist $[15,16]$, which cannot be investigated by means of other techniques. Indeed, standard solution procedures for flow equations rely on relaxation methods (which need fine-tuned initial conditions in presence of multiple fixed points), polynomial expansions (which assume analytic form for the effective potential) or full scale dependent solution (which need approximate knowledge of the final form for the effective potential). In contrast our numerical 
procedure is unbiased and does not assume any further knowledge of the theory under consideration apart for its global symmetry, neither it introduces further approximation rather than the EEA ansatz necessary to derive the flow equations.

\section{DERIVATIVE EXPANSION}

The derivative expansion is an approximation scheme where the EAA is expanded in powers of the field spatial derivatives $[2,3,17,18]$. This scheme is usually employed for matter field theories on flat space, where it becomes a series expansion in powers of the momentum. The derivative expansion has been very successful in drawing phase diagrams and computing accurate universal quantities, especially critical exponents.

If one considers a one-component scalar field, in $d$-dimensional flat space, with a $\mathbb{Z}_{2}$ symmetry, the derivative expansion for the EAA to order $O\left(\partial^{2}\right)$ reads

$\Gamma_{k}[\phi]=\int d^{d} x\left\{V_{k}(\phi)+\frac{1}{2} Z_{k}(\phi)(\partial \phi)^{2}\right\}+O\left(\partial^{4}\right)$.

The effective potential $V_{k}(\phi)$ and the wave-function renormalization function $Z_{k}(\phi)$ are arbitrary functions of the field $\phi$. The derivative expansion has been carried to higher order in $d=3$ [17], where a beta function study was performed. The flow equations for these functions are derived from the exact RG equation satisfied by the EAA $[2,3]$,

$$
\partial_{t} \Gamma_{k}[\phi]=\frac{1}{2} \operatorname{Tr}\left(\Gamma_{k}^{(2)}[\phi]+R_{k}\right)^{-1} \partial_{t} R_{k} .
$$

For translational invariant systems the trace in latter equation represents a momentum integral and the cutoff function $R_{k}$ is a momentum-dependent mass term introduced into the effective action in order to freeze the low energy excitations responsible for the appearance of infrared (IR) divergences. The effective action explicitly depends on a scale $k$ and the renormalization "time" is defined as $t=\log \left(k / k_{0}\right)$, where $k_{0}$ is an arbitrary reference scale.

After inserting the ansatz (1) into the flow equation (2) and performing the appropriate projection one can derive the beta functionals $\beta_{V}$ and $\beta_{Z}$ for the running effective potential and field-dependent wave-function renormalization function

$$
\begin{gathered}
\partial_{t} V_{k}=\beta_{V}^{d}\left(V_{k}^{\prime \prime}, Z_{k}\right) \\
\partial_{t} Z_{k}=\beta_{Z}^{d}\left(V_{k}^{\prime \prime}, V_{k}^{\prime \prime \prime}, Z_{k}, Z_{k}^{\prime}, Z_{k}^{\prime \prime}\right) .
\end{gathered}
$$

The explicit form of the beta functionals can be obtained in arbitrary dimension and for arbitrary cutoff functions. The beta functional for the effective potential follows directly by evaluating (2) at a constant field configuration and reads

$$
\beta_{V}^{d}=\frac{1}{(4 \pi)^{\frac{d}{2}}} \frac{1}{2} Q_{\frac{d}{2}}\left[G_{k} \partial_{t} R_{k}\right]
$$

In (4) the regularized propagator at the constant field configuration $\phi$ is defined as

$$
G_{k}(x, \phi)=\frac{1}{Z_{k}(\phi) x+V_{k}^{\prime \prime}(\phi)+R_{k}(x)},
$$

while the $Q$ functionals are defined as

$$
Q_{n}[f] \equiv \frac{1}{\Gamma(n)} \int_{0}^{\infty} \mathrm{d} x x^{n-1} f(x) .
$$

Deriving the flow equation for the wave-function renormalization function is more involved. Taking the second functional derivative of (2) with respect to the fields it is possible to write down the flow equation for the two-point function of the EAA in momentum space. Introducing on the rhs of this equation the vertices of the EAA (1) and extracting the $O\left(p^{2}\right)$ terms one obtains, after some algebra, the following beta functional for the wave-function renormalization function

$$
\begin{aligned}
\beta_{Z}^{d}= & \frac{\left(V_{k}^{\prime \prime \prime}\right)^{2}}{(4 \pi)^{\frac{d}{2}}}\left\{Q_{\frac{d}{2}}\left[G_{k}^{2} G_{k}^{\prime} \partial_{t} R_{k}\right]+Q_{\frac{d}{2}+1}\left[G_{k}^{2} G_{k}^{\prime \prime} \partial_{t} R_{k}\right]\right\}+\frac{\left(Z_{k}^{\prime}\right)^{2}}{(4 \pi)^{\frac{d}{2}}}\left\{\frac{2 d+1}{2} Q_{\frac{d}{2}+1}\left[G_{k}^{3} \partial_{t} R_{k}\right]+\frac{(d+2)(d+4)}{4}\right. \\
& \left.\times\left(Q_{\frac{d}{2}+2}\left[G_{k}^{2} G_{k}^{\prime} \partial_{t} R_{k}\right]+Q_{\frac{d}{2}+3}\left[G_{k}^{2} G_{k}^{\prime \prime} \partial_{t} R_{k}\right]\right)\right\}+\frac{V_{k}^{\prime \prime \prime} Z_{k}^{\prime}}{(4 \pi)^{\frac{d}{2}}}\left\{2 Q_{\frac{d}{2}}\left[G_{k}^{3} \partial_{t} R_{k}\right]+(d+2)\left(Q_{\frac{d}{2}+1}\left[G_{k}^{2} G_{k}^{\prime} \partial_{t} R_{k}\right]\right.\right. \\
& \left.\left.+Q_{\frac{d}{2}+2}\left[G_{k}^{2} G_{k}^{\prime \prime} \partial_{t} R_{k}\right]\right)\right\}+\frac{Z_{k}^{\prime \prime}}{(4 \pi)^{\frac{d}{2}}}\left\{-\frac{1}{2} Q_{\frac{d}{2}}\left[G_{k}^{2} \partial_{t} R_{k}\right]\right\} .
\end{aligned}
$$

Equations (4) and (7) represent the flow equations for $V_{k}(\phi)$ and $Z_{k}(\phi)$ for general cutoff function at $O\left(\partial^{2}\right)$ of the derivative expansion. Once an appropriate cutoff shape has been chosen, the integrals in (4) and (7) can be performed. In this way one obtains a system of partial differential equations for $V_{k}(\phi)$ and $Z_{k}(\phi)$ in the variables $k$ and $\phi$. 
Finally the flow equations are obtained introducing the dimensionless variables. The dimensionless quantities are defined as

$$
\begin{gathered}
\phi=Z_{k}^{-\frac{1}{2}} k^{\left(\frac{d}{2}-1\right)} \varphi, \quad V_{k}(\phi)=k^{d} v(\varphi), \\
Z_{k}(\phi)=Z_{k} \zeta(\varphi),
\end{gathered}
$$

from which we drive the following relations by $t$ differentiation

$$
\begin{aligned}
& \beta_{v}=-d v+\frac{d-2+\eta}{2} \varphi v^{\prime}+k^{-d} \beta_{V} \\
& \beta_{\zeta}=\eta \zeta+\frac{d-2+\eta}{2} \varphi \zeta^{\prime}+Z_{k}^{-1} \beta_{Z},
\end{aligned}
$$

where $\beta_{v} \equiv \partial_{t} v$ and $\beta_{\zeta} \equiv \partial_{t} \zeta$. The anomalous dimension of the scalar field in (8) is defined by $\eta=-\partial_{t} \log Z_{k}(0)$ [4].

To obtain explicit expressions for the beta functional, we need to specify the cutoff function $R_{k}$. In terms of the linear cutoff $^{1}$ introduced in [19],

$$
R_{k}(x)=Z_{k}\left(k^{2}-x\right) \theta\left(k^{2}-x\right),
$$

the $Q$ functionals in (4) and (7) can all be computed analytically and they can be reduced to a single threshold integral, a hypergeometric function (see the Appendix A). In integer dimension, explicit expressions for $\beta_{v}$ and $\beta_{\zeta}$ can be written, as reported in the Appendix $\mathrm{C}$.

\section{SCALING SOLUTIONS}

In this formalism the scaling solutions for the effective actions appear as FRG fixed points and they are determined by solving the coupled system of ordinary differential equations

$$
\beta_{v}=0 \quad \beta_{\zeta}=0
$$

For parity reasons we expect the following boundary conditions for the effective potential and the wave-function renormalization functional evaluated at the origin,

$$
\begin{gathered}
v^{\prime}(0)=0 \\
v^{\prime \prime}(0)=\sigma \\
\zeta(0)=1 \\
\zeta^{\prime}(0)=0 .
\end{gathered}
$$

\footnotetext{
${ }^{1}$ The linear cutoff is believed to be the optimal choice at low order in derivative expansion [19]. Indeed the results for the critical exponents found in this case are more accurate than the ones obtained with power law cutoff $[3,11]$, as discussed in the following.
}

Conditions (11) and (14) are a direct consequence of the $\mathbb{Z}_{2}$ symmetry. The condition (14) is obtained absorbing a factor $Z_{k}(0)$ into the field redefinition. Thus the only unspecified condition remaining is the $v^{\prime \prime}(0)$ value. In Eq. (12) $\sigma$ and $\eta$ are real values to be determined by requiring the global existence of the scaling solutions, condition that, as we will see, reduces to a finite set the allowed functional fixed points.

The main goal of our paper is to show that it is possible to find solutions of the system (10) extending the simple spike plot technique used in LPA analysis $[7,10]$. This method has diverse advantages: first of all it does not require the solution of the flow equations in function of the renormalization time $t$; second it solves the fixed point equations in their full functional form, without relying on truncations [17] (this property is necessary to be consistent with the Mermin-Wagner theorem [20]); third it does not necessitate any external input as in $[3,11]$, where the relaxation method was used starting from the exact spherical model solution. For these reasons the present method is the most suited to uncover actual nonperturbative universality classes, which can not be investigated by means of standard approaches [15].

Let us describe the procedure in more details, we solve Eq. (10) for different values of $\sigma$ and $\eta$. For any arbitrary point in the $(\sigma, \eta)$ plane the solution will become singular at a finite value of the field [21], we call this finite value $\varphi_{\text {sing, }}$, and global scaling solutions correspond to those points in which $\varphi_{\text {sing }}$ shows a "spike" behaviour. Thus physical fixed points in the $(\sigma, \eta)$ plane can be determined by a numerical analysis of the function $\varphi_{\text {sing }}$.

For the purpose of extending the resulting scaling solutions $v$ and $\zeta$ beyond $\varphi_{\text {sing }}$ the analysis is complemented by the asymptotic behaviours computed using the large field solutions of Eq. (10)

$$
\left\{\begin{array}{l}
v(\varphi) \sim v_{0} \varphi^{\frac{2 d}{d-2+\eta}} \\
\zeta(\varphi) \sim \zeta_{0} \varphi^{-\frac{2 \eta}{d-2+\eta}}
\end{array} \text { for } \varphi \gg 1 .\right.
$$

Once the location of a fixed point in the $(\sigma, \eta)$ plane has been determined, the coefficients $\left(v_{0}, \zeta_{0}\right)$ can be evaluated by imposing continuity of the numerical solutions for $v$ and $\zeta$ with the large field expansion in Eq, (15) at some value $\varphi_{\text {max }}<\varphi_{\text {sing }}$.

We will show in subsection III A that, in $d=3$, there is only one scaling solution to the system (10), which corresponds to the so-called Wilson-Fisher fixed point and describes the Ising $d=3$ universality class. This fixed point was extensively studied in the literature, also with shooting techniques similar to the one discussed in this paper $[14,22,23]$. It is in $d=2$, where every perturbative approach fails to describe correctly the various universality classes, first constructed exactly using conformal field theory (CFT) methods [24], that the system (10) reveals 
its nonperturbative potentialities. It was shown in [3] that the power-law cutoff version of the system (10), solved with the relaxation method [25], has scaling solutions in one-to-one correspondence with the minimal models known from CFT. In subsection III B, we will see that the same picture emerges also when the linear cutoff and the spike plot method are employed.

\section{A. The three-dimensional case}

First of all we consider the $d=3$ case, which is the less involved since only the standard Wilson-Fisher fixed point exists. The equations for the $\beta$ functions in the linear regulator case, $(\mathrm{C} 3)$ and $(\mathrm{C} 4)$ from the Appendix, are set to zero and solved numerically, with a fourth-order RungeKutta approach, for several points in the $(\sigma, \eta)$ plane. The value at which the numerical solver interrupts integration $\varphi_{\text {sing }}$ is represented as a contourplot in the plane of the initial conditions $(\sigma, \eta)$ in Fig. 1 panel (a).

Apart for the maximum in the origin, which represents the free Gaussian universality class, we see a family of maxima arising along a curve which separates the dark blue region of the severely ill-defined solutions from the light green region of intermediate solutions. Only a small portion of the plane has a nontrivial support for the $\varphi_{\text {sing }}$ function, which is identically zero for all $\sigma<-0.2$. Moreover the equations in the linear cutoff case develop a divergence for $\sigma=-1$ often called spinodal instability. It is not clear wether this divergence, which is IR attractive in all directions, is the physical IR fixed point or rather an artifact of the linear regulator scheme. However it has been demonstrated that it is possible to recover information over the universal quantities studying the scaling of the solutions close to this instability [26].

The landscape in Fig. 1 can seem surprising due to the presence of several maxima rather than an isolated one, indicating the Ising universality class. However a closer inspection reveals that the high of the peaks is nonmonotonous, leading to the appearance of a prominent maximum at finite $\eta$ and $\sigma$ values. This prominent maximum represents the signature of the Wilson-Fisher fixed point. The connection of the Ising and Gaussian universalities through an extended chain is easily justified if one considers that the spike-plot technique furnishes a solution even in the lower truncation scheme, where only the potential equation is considered. Thus it appears that for each value of $\eta$ it exists a maximum as a function of $\sigma$ which represents the best approximation for the $v(\varphi)$ and $\zeta(\varphi)$ functions at that particular value of the anomalous dimension.

On the other hand, due to the finite numerical grid in the $(\sigma, \eta)$ plane, to the complex three-dimensional shape of the chain as well as to the finite numerical accuracy in the solution of the flow equations, the peaks chain appears as a sequence of spikes rather than a continuous ridge. This picture is confirmed in the following, where we pursue the

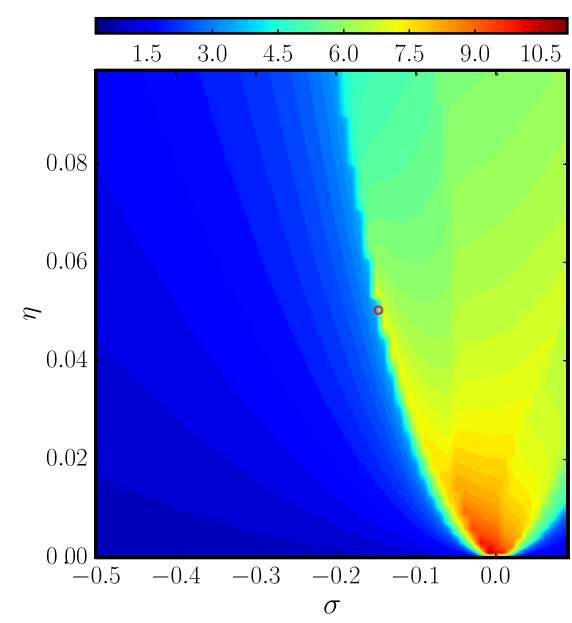

(a) Contour plot of $\varphi_{\operatorname{sing}}(\sigma, \eta)$ in $d=3$

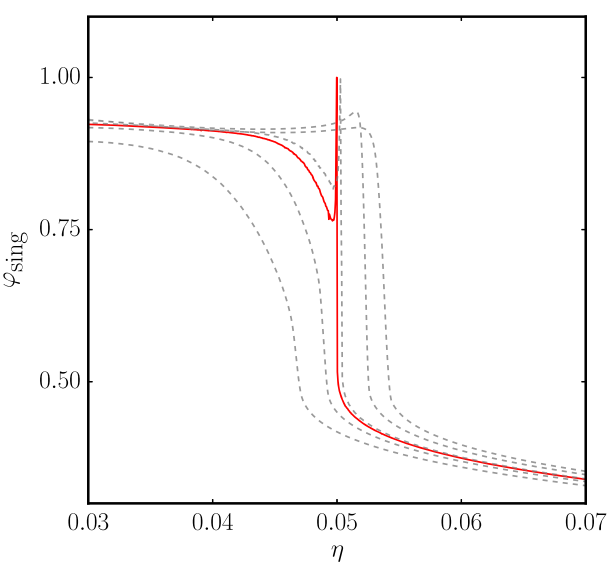

(b) Spike-plot along the chain of maxima in the contour plot of panel (a).

FIG. 1. The contour plot of $\varphi_{\text {sing }}$ in the $(\sigma, \eta)$ planes has two spikes: the one located at $(0,0)$ represents the Gauss i an theory. The other one indicates the Ising universality class, which occurs at a finite positive value of the anomalous dimension and for a negative dimensionless mass. In panel (b), the spike-plot is performed along the maxima chain of the contour plot of panel (a) with different choices for the fit parameters (gray dashed lines). The optimal choice (red solid line) is the one which maximizes the spike height, see the main text.

analysis along several trajectory in the $(\sigma, \eta)$, retrieving the expected scenario. As anticipated above, a convenient approach to overcome these difficulties and to extract the anomalous dimension of the interacting universality classes is to reduce the problem of finding the maxima of the two-dimensional surface $\varphi_{\text {sing }}(\sigma, \eta)$ to the simpler one of locating the maximum in a single one-dimensional chain. This is possible using the results in panels (a) of Figs. 1 and 3 as a guide.

First of all, we fit the locations of the maxima of a single chain in the $(\sigma, \eta)$ plane with a simple function; depending on the case, linear or parabolic fit functions are employed. This operation produces an explicit expression for the 


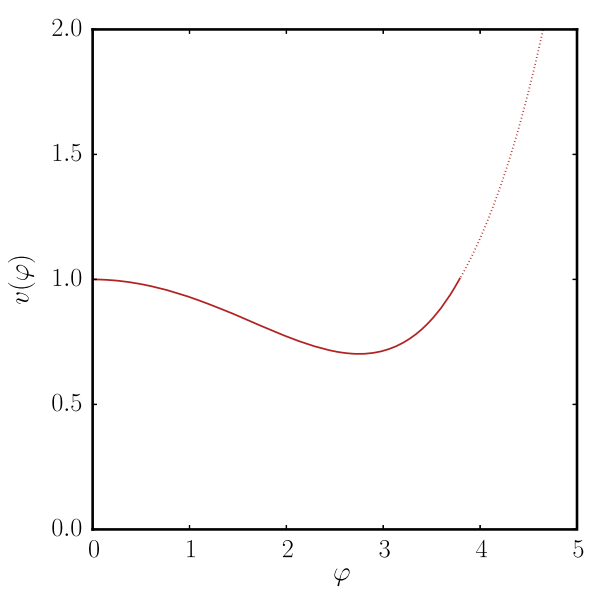

(a) Fixed point potential $v(\varphi)$ in $d=3$

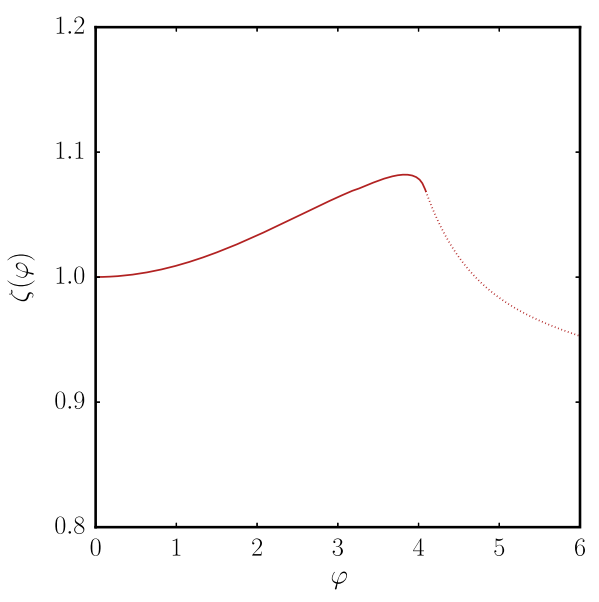

(b) Fixed point wave-function $\zeta(\varphi)$ in $d=3$

FIG. 2. In panels (a) and (b), respectively, the solutions for the functions $\zeta(\varphi)$ and $v(\varphi)$ are shown for the Wilson-Fisher fixed point in three dimension. The lines have been obtained imposing continuity between the numerical solution (solid line) and analytic large field behaviour of the scaling solutions (15) (dotted lines).

maxima chain as a function $\sigma(\eta)$ along which we can pursue the standard one-dimensional spike plot technique. Obviously the coefficients of the fit will contain errors due to the finiteness of the grid in the landscapes of panels (a) in Figs. 1 and 3. However the best value for the fit is obtained optimizing the coefficient in order to maximize the height of the spike; as it is shown in panel (b) of Fig. 1 for the three-dimensional Ising universality. The optimization procedure is straightforward since one should allow only for very small variation of the fit coefficients and the onedimensional spike plot computation is extremely fast.

In the three-dimensional case, the optimization procedure is shown in Fig. 1 panel (b). The grey dashed curves are the spike plots along different fit representations of the peak chain in panel (a), Fig. 1. The equation for the peak chain has been obtained by linear fit around the position of the nontrivial maximum. The various curves (gray dashed lines) show the result for different values of the fit coefficients, the optimized curve, red solid line, gives $\eta=0.0496$ with $\sigma=-0.1468$. The fixed point potential and wave-function renormalization are shown in Fig. 2 panel (a) and (b) respectively. In correspondence of the minimum of the fixed point potential, a maximum of the wave function is found. The large field behavior can not be obtained by numerical integration, since a very precise identification of the correct fixed point values $(\sigma, \eta)$ is necessary to push the integration forward. In Fig. 2, the large field branch of the two functions has been obtained using large field expansions reported in Eq. (15) and imposing continuity with the numerical solutions.

The numerical value for the critical exponent $\eta=0.0496$ is in better agreement with exact Monte Carlo (MC) and conformal bootstrap results $[27,28] \eta=0.0363$, with respect to the power law regulator results present in literature $\eta=0.0539$ [14], in agreement with the expectation for the linear regulator of being the optimal cutoff for derivative expansions.

\section{B. The two-dimensional case}

In the $d=2$ case, scalar $\mathbb{Z}_{2}$ theories have an infinite number of universality classes, leading to a far more complicated landscape in the $(\sigma, \eta)$ plane. These universality classes correspond with the minimal models of CFT and the critical exponents are known exactly. The importance of reproducing such results in an approximated scheme is not only the methodological one, deriving from the necessity to test our technique in a more complex scenario, but it also lies in the different assumptions necessary to compute such quantities. Indeed, flow equations (4) and (7) have been derived without any additional condition rather than $\mathbb{Z}_{2}$ symmetry. Even in this oversimplified computation scheme and without any additional imposition on the symmetry of the model, such as conformal symmetry, the FRG technique is able to recover all the information on the location and the shape of the solutions, with a good numerical accuracy on the universal quantities.

The result for the landscape of interacting fixed points in two-dimensional scalar field theories is reported in Fig. 3, in the linear cutoff scheme. Identifying correctly the exact value of the anomalous dimension for any universality class requires some care. Indeed, similarly to the $d=3$ case in Fig. 1, the maxima of $\varphi_{\text {sing }}$ are disposed on special lines of the $(\sigma, \eta)$ plane, forming peak chains. In panel (a) of Fig. 3, the Gaussian universality class appears as a infinitely tall spike located at $\sigma=\eta=0$. The two peaks chains at the extrema of the origin are the longest in the $\eta$ direction and they represent the Wilson-Fisher and tricritical universalities which have the largest anomalous dimension values. Both these peak chains, going from $\eta=0$ to $\eta \simeq 1$, have a maxima at a finite value of the anomalous dimension which roughly corresponds to the expected one. Moreover we 


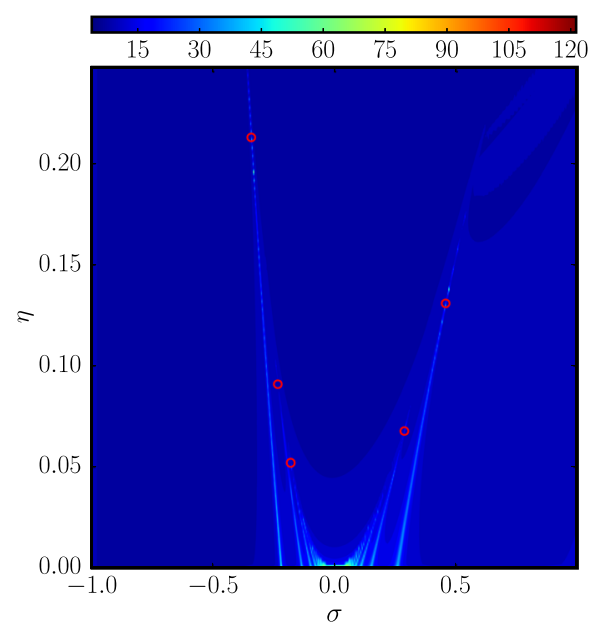

(a) Contour plot of $\varphi_{\text {sing }}(\sigma, \eta)$ in $d=2$

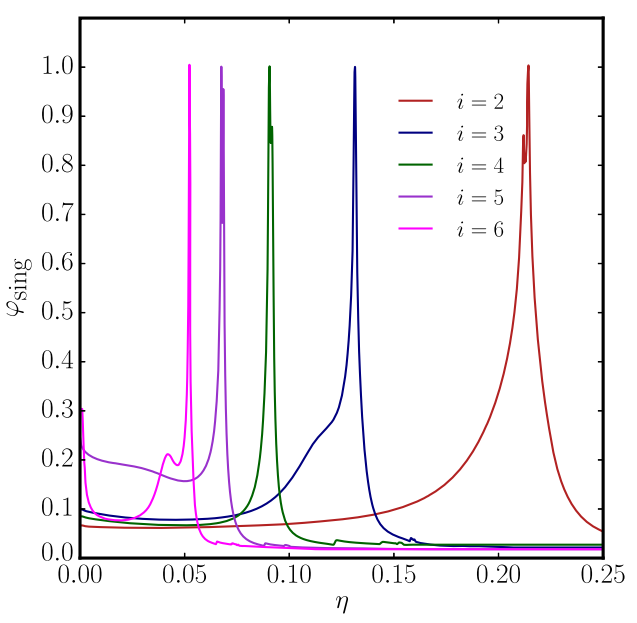

(b) Spike plots along the chains of panel (a)

FIG. 3. In panel (a), spike plot in two dimensions for the linear regulator case. The landscape of scalar quantum field theories at this approximation level is quite complicated. The maxima of $\varphi_{\text {sing }}$ form mountain chains located on some special curves in the $(\sigma, \eta)$ plane.

have infinitely many other peak chains starting at $\eta=0$. These chains accumulate in the origin, becoming shorter and shorter, as expected since they represent high-order universalities.

As for the three-dimensional case, the finite numerical grid in the $(\sigma, \eta)$ plane does not allow for a precise location of the tallest maximum along each chain. Increasing the precision of the grid will need an exponential growing computation time due to the necessity to increase accordingly the precision of the numerical solution of the differential equations. Once again, in order to maintain a low computational cost, we consider a numerical fit for each peak chain along which we pursue a one-dimensional spike plot procedure analogue to the LPA case. Optimizing the fit coefficients to maximize the height of the peak in every chain of the contour plot in $d=2$, Fig. 3 panel (a), we

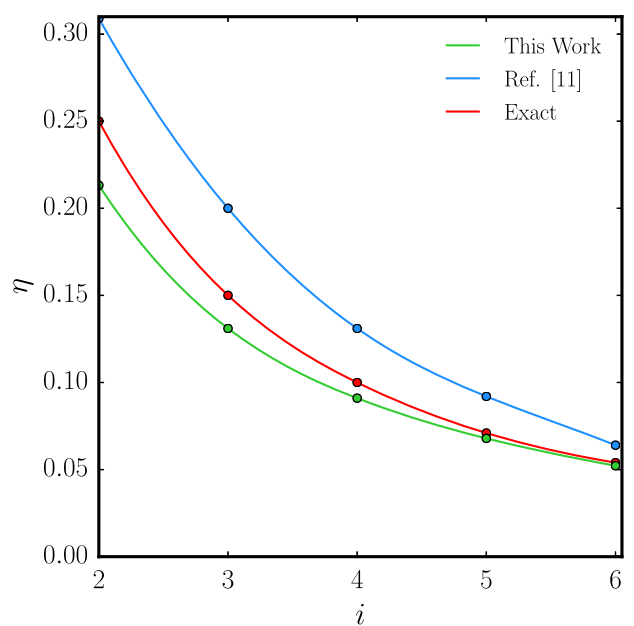

(a) Anomalous dimensions of the first six Multi-critical universality classes in $d=2$
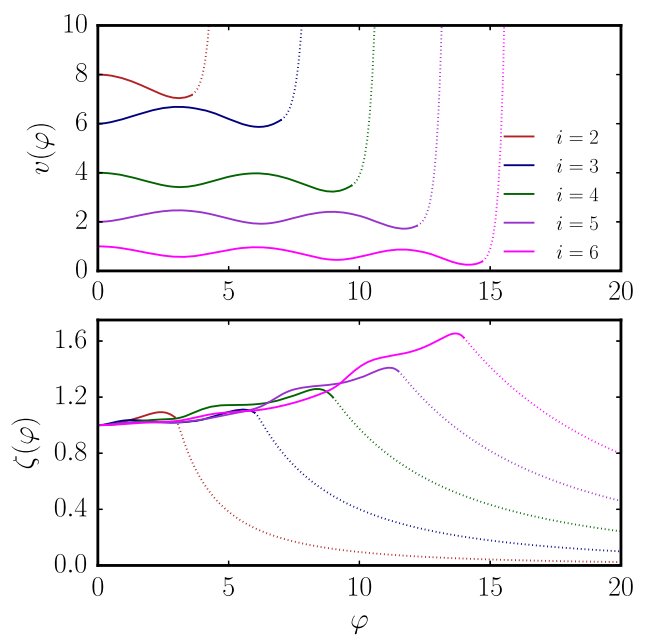

(b) Fixed point solutions for the functions $v(\varphi)$ and $\zeta(\varphi)$ in $d=2$

FIG. 4. In panel (a), we show the anomalous dimensions as a function of the critical index $i$ for the linear (green line) and power law (blue line) cutoff case, compared to the exact CFT results (red line). In panel (b), the solutions for the functions $\zeta(\varphi)$ and $v(\varphi)$ are shown for the first six multicritical universalities. Each line has been obtained imposing continuity between the numerical solution (solid line) and analytic large field behaviour of the scaling solutions (15) (dotted lines).

obtain a one-dimensional spike plot for every universality class from the standard Wilson-Fisher case $i=2$ to the esacritical universality $i=6$ as it is shown in Fig. 3 panel (b). The values, at which the singularities of $\varphi_{\text {sing }}$ occur, are in agreement with the expected values for the anomalous dimensions. During the optimization procedure of the fit parameters it occurs that two minima are found in the line, proving the complex three-dimensional structure of the peaks in the $(\sigma, \eta)$ plane. In any case, the highest divergence is always obtained for a single peak, confirming that the two peaks structure occurs only when the fit line does not cross the spike at its center. The distance between the two 
peaks can be easily reduced until a precision $\leq 5 \%$ is obtained in the $\eta$ values, see Fig. 3 panel (b).

In Fig. 4, panel (a), the anomalous dimension values obtained using the spike plot technique are shown. The results of this work (green line) are compared to the ones found in [11] (blue line) and to the exact results of CFT solutions (red line). The curve obtained here is more precise than the results found in the power law cutoff scheme of [11], which confirms the expected better performance of the linear cutoff scheme. It should be also noted that, as expected, the precision of FRG truncation scheme increases lowering the anomalous dimension values, with the esacritical value for the $\eta$ exponent reproduced up to $99 \%$ even in this rather simple approximation. The fact that the precision of FRG truncation scheme increases lowering the anomalous dimension values suggests that a truncation based on the real fixed point dimension of the operators included in the EAA might be better than the derivative expansion from the quantitative point of view. While in $d=2$ for $\eta=0$, the expansion in terms of the operator dimensions and derivative expansion coincides as $\eta \rightarrow 0$ and, thus, as $\eta$ grows, they start diverging. In $d=3$, the situation is very different and may explain the less precise numerical success of the derivative expansion in this dimension.

In panel (b) of Fig. 4, we show the potential $v(\varphi)$ and the field-dependent wave function renormalization $\zeta(\varphi)$ for the first six universality classes in $d=2$. The solutions are shown only for positive values of the field $\varphi$, since the other branch can be simply obtained using reflection symmetry. Each potential shows a number of minima $i$, as indicated by its criticality order, and the corresponding wave function renormalization has a relative maximum in correspondence of each minimum position. The solutions shown in Fig. 4 panel (b) have been obtained solving Eqs. (4) and (7) with the values of $\eta$ and $\sigma$ found using the spike plot technique described in Fig. 1 panel (b).

\section{Regulator dependence}

As it should be understood from the above investigations, the efficiency of our spike plot technique crucially depends on the structure of the three-dimensional surface $\varphi_{\text {sing }}(\sigma, \eta)$, which has nontrivial shape only on a finite number of quasi-two-dimensional manifolds, i.e., the lines in Fig. 3. It is then necessary to test whether this simplified structure is just an artifact of the linear cutoff function or if it is a general result valid for Eqs. (4) and (7) independently from the particular form assumed by the $Q$ functionals.

In order to check the stability of our approach, we consider another cutoff function to explicitly compute the $Q$ functionals. The most effective choice in this perspective is the power law regulator already adopted in [11] $R_{k}(x)=k^{4} / x$. Indeed, such a regulator, even if not optimal to compute numerical quantities, produces simple results for the flow equations. Moreover the power law cutoff has a

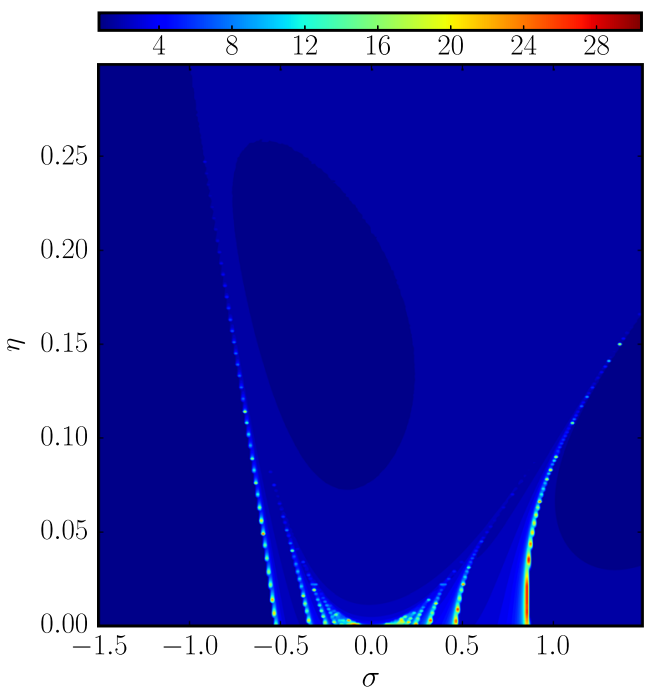

(a) Contour plot of $\varphi_{\operatorname{sing}}(\sigma, \eta)$ in $d=2$ for the power law regulator

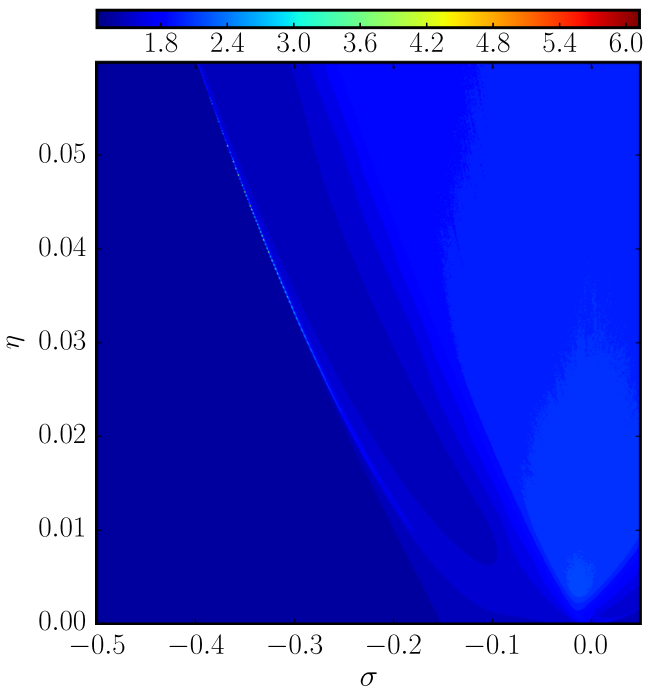

(b) Contour plot of $\varphi_{\text {sing }}(\sigma, \eta)$ in $d=3$ for the power law regulator

FIG. 5. Spike plot in two dimensions for the power law cutoff case. The landscape of scalar quantum field theories at this approximation level is quite complicated. The maxima of $\varphi_{\text {sing }}$ form mountain chains located on some special curves in the $(\sigma, \eta)$ plane.

rather peculiar shape, completely different from the linear cutoff employed above. Indeed, while the last one is compact with extremely localized derivatives the first one has infinite support and it has finite derivatives at all orders.

We apply the same procedure already considered for the flow equations in the linear regulator case. Both in $d=2$ and $d=3$ we retrieve the expected phase structure, with only one correlated fixed point in the first case and infinitely many solutions in $d=2$, as shown in Fig. 5. It is worth noting that in the power law regulator case the height of the peaks in the contour plot is much smaller than 
in the previous case. However both in 2, Fig. 5 panel (a), and 3, panel (b), the peak chains are evident, thus demonstrating that the structure of the $\varphi_{\text {sing }}(\sigma, \eta)$ function, even if influenced by the regulator form, maintains a very small nontrivial support. In both the regulator cases, every universality class of the theory appears as a chain of peaks in the $(\sigma, \eta)$ plane.

\section{CONCLUSION}

After deriving the explicit expressions for the flow equations of the effective potential $V_{k}$ and the wavefunction renormalization function $Z_{k}$, in two and three dimensions, and with the use of the linear cutoff, we have shown that it is possible to extend the "spike plot" technique to the study of scaling solutions at order $\partial^{2}$ of the derivative expansion. In this approximation, theory space is projected to the two-dimensional plane parametrized by $\sigma \equiv v^{\prime \prime}(0)$ and by the anomalous dimension $\eta$. The spike plot becomes a two-dimensional surface that represents the landscape of scalar theories with $\mathbb{Z}_{2}$ symmetry. These landscapes are characterized by "peak chains" along which the spikes appear; the shape of these chains can be fitted by a simple linear or quadratic curve $\sigma(\eta)$ and the problem of finding the position of the maxima of these surfaces can be reduced to a one-dimensional spike plot similar to those found at the LPA level.

In $d=3$, the landscape is characterized by a single nontrivial spike that represents the Ising universality class, while in two dimensions the landscape is much more complex: a whole chain is present for each Multicritical universality class, and each CFT minimal model emerges as the most prominent peak of each of these chains. At high multicriticality, the obtained values of the anomalous dimensions are in very good agreement with the exact CFT results. At the quantitative level, the increasing precision with which the anomalous dimension of the multicritical fixed points is obtained, for growing multicriticality index, suggests that an expansion scheme based on the relevancy of the real operator dimension (operator dimension expansion) of the terms included in the truncation ansatz of the effective average action might be quantitatively precise. This is suggested by the fact that the derivative expansion, in $d=2$ and as $\eta \rightarrow 0$, becomes exactly the operator dimension expansion and indeed we obtain better and better values for $\eta$ as the multicriticality grows.

While the present analysis shows how the spike plot technique can produce a complete and systematic analysis of fixed point solutions of functional flow equations, even beyond the well-known LPA case, it would be interesting to disclose the full picture of the critical exponents for scalar field theories, including the correlation length exponent $\nu$, even for fractional dimension beyond two and three where it will be interesting to compare with the recent $\epsilon$-expansion analysis [29-32]. However the latter task is hindered by the complexity of the flow equations, even in the case of the linear regulator (9), which leads to substantial numerical difficulties, especially in the study of the stability exponents. Then, it would be more convenient to consider different regularization schemes which deliver considerably simpler functional flow equations [33]. Another interesting perspective is the study of the nonunitary family of fixed points generalizing the LPA analysis [34] or long range interacting field theories, both in the classical [35] and quantum case $[36,37]$ or, finally in presence of global symmetries as $O(N)$ interactions $[8,9,15]$ or Potts model $S_{n+1}$-symmetries [38]. We leave all these applications to future works.

Since the spike plot method does not need any prerequisites and avoids further approximations apart from the derivative expansion itself, it is the most suited technique to uncover strongly nonperturbative aspects of critical phenomena. To this aim the generalization of the present technique to larger sets of flow equations will be necessary, leading to an enlarged parameter space for the initial value problem. However, we expect the physical solutions to always lie on low dimensional subsets of the parameter space as it happens in the present case, see panels (a) in Figs. 3 and 1. Once these relevant regions have been identified, looking for the maxima of $\varphi_{\text {sing }}$ on a rough grid, the investigation can be refined by constraining the parameters to lie in the relevant regions. Afterwards, the constraints shall be optimized by magnifying the singularity of $\varphi_{\text {sing }}$, as it is shown in Fig. 1 panel (b). The procedure in an higherdimensional space will thus be analogue to the one described in the present paper and it could lead to the identification and description of novel universality classes, whose existence is, till now, doubted $[39,40]$.

\section{AKNOWLEDGMENTS}

We would like to thank M. Safari for his help and collaboration in the early stages of this work. N. D. acknowledges support by the Deutsche Forschungsgemeinschaft Collaborative Research Centre "SFB 1225 (ISOQUANT)."

\section{APPENDIX A: DERIVATION OF $\partial_{t} V_{k}$ AND $\partial_{t} Z_{k}$}

The effective average action to the second order in the derivative expansion is given by

$$
\Gamma_{k}=\int_{x}\left(\frac{1}{2} Z(\varphi) \partial_{\mu} \varphi \partial^{\mu} \varphi+V(\varphi)\right),
$$

where $V(\varphi)$ is the effective potential and $Z(\varphi)$ is the generalized wave function renormalization. The functional derivatives of the action (A1) in momentum space for a constant field configuration read

$$
\begin{aligned}
\Gamma_{q_{1} q_{2}}^{(2)}= & (2 \pi)^{d}\left(-Z q_{1} \cdot q_{2}+V^{(2)}\right) \delta\left(q_{1}+q_{2}\right) . \\
\Gamma_{q_{1} q_{2} q_{3}}^{(3)}= & (2 \pi)^{d}\left[-Z^{(1)}\left(q_{1} \cdot q_{2}+q_{1} \cdot q_{3}+q_{2} \cdot q_{3}\right)+V^{(3)}\right] \\
& \times \delta\left(q_{1}+q_{2}+q_{3}\right),
\end{aligned}
$$




$$
\begin{aligned}
\Gamma_{q_{1} q_{2} q_{3} q_{4}}^{(4)}= & (2 \pi)^{d}\left[-Z^{(2)}\left(q_{1} \cdot q_{2}+q_{1} \cdot q_{3}+q_{1} \cdot q_{4}\right.\right. \\
& \left.\left.+q_{2} \cdot q_{3}+q_{2} \cdot q_{4}+q_{3} \cdot q_{4}\right)+V^{(4)}\right] \\
& \times \delta\left(q_{1}+q_{2}+q_{3}+q_{4}\right) .
\end{aligned}
$$

The flow equations for the potential is

$$
\partial_{t} V_{t}=\frac{1}{2} \int \frac{d^{d} q}{(2 \pi)^{d}} G(q) R_{k}(q),
$$

and for the two point function in the standard form is

$$
\begin{aligned}
\partial_{t} \Gamma_{t, p,-p}^{(2)}= & \int d^{d} q G(q) \Gamma_{q, p,-q-p}^{(3)} G(q+p) \\
& \times \Gamma_{q+p,-p,-q}^{(3)} G(q) \partial_{t} R_{k}(q) \\
& -\frac{1}{2} \int d^{d} q G(q) \Gamma_{q, p,-p,-q}^{(4)} G(q) \partial_{t} R_{k}(q),
\end{aligned}
$$

where the short hands are obvious. We have

$$
\begin{aligned}
\Gamma_{q, p,-p,-q}^{(4)} & =Z^{(2)}\left(q^{2}+p^{2}\right)+V^{(4)} \\
\Gamma_{q, p,-q-p}^{(3)} & =Z^{(1)}\left(q^{2}+q \cdot p+p^{2}\right)+V^{(3)} \\
\Gamma_{q+p,-p,-q}^{(3)} & =Z^{(1)}\left(q^{2}+q \cdot p+p^{2}\right)+V^{(3)} .
\end{aligned}
$$

In order to evaluate the flow of the field-dependent wave function, we use the definition

$$
\partial_{t} Z(\varphi)=\frac{1}{2} \lim _{p \rightarrow 0} \frac{d^{2}}{d p^{2}} \partial_{t} \Gamma_{t}^{(2)}(-p, p) .
$$

When we apply the derivatives on the right end side of Eq. (A6), they go under the integral sign and act in the only part of the integrand which depends on $p$, i.e., $G(p+q)$; thus, we get

$$
\begin{aligned}
\partial_{t} Z(\varphi)= & \frac{1}{2} \int \frac{d^{d} q}{(2 \pi)^{d}} \partial_{t} R_{t}(q) G(q)^{2} \Gamma_{q, p,-p-q}^{(3)} \frac{d^{2}}{d p^{2}} \\
& \times\left. G(p+q) \Gamma_{-q,-p, p+q}^{(3)}\right|_{p=0} \quad(\mathrm{~A} 11) \\
& +\int \frac{d^{d} q}{(2 \pi)^{d}} \partial_{t} R_{t}(q) G(q)^{2} \Gamma_{q, p,-p-q}^{(3)} G(p+q) \frac{d^{2}}{d p^{2}} \\
& \times\left.\Gamma_{-q,-p, p+q}^{(3)}\right|_{p=0} \quad(\mathrm{~A} 12) \\
& +4 \int \frac{d^{d} q}{(2 \pi)^{d}} \partial_{t} R_{t}(q) G(q)^{2} \Gamma_{q, p,-p-q}^{(3)} \frac{d}{d p} \\
& \times\left. G(p+q) \frac{d}{d p} \Gamma_{-q,-p, p+q}^{(3)}\right|_{p=0} \\
& +\int \frac{d^{d} q}{(2 \pi)^{d}} \partial_{t} R_{t}(q) G(q)^{2} G(p+q) \\
& \times\left.\left(\frac{d}{d p} \Gamma_{-q,-p, p+q}^{(3)}\right)^{2}\right|_{p=0} \\
& -\left.\frac{1}{4} \int d^{d} q G(q) \frac{d^{2}}{d p^{2}} \Gamma_{q, p,-p,-q}^{(4)} G(q) \partial_{t} R_{k}(q)\right|_{p=0}
\end{aligned}
$$

The calculation is straightforward but complex, we shall then carry it out term by term. We then define the first term $T_{1}$ as the right hand side of (A11)

$$
\begin{aligned}
T_{1}= & \int \frac{d^{d} q}{(2 \pi)^{d}} \partial_{t} R_{t}(q) G(q)^{2} \Gamma_{q, p,-p-q}^{(3)} \frac{1}{2} \frac{d^{2}}{d p^{2}} \\
& \times\left. G(p+q) \Gamma_{-q,-p, p+q}^{(3)}\right|_{p=0}
\end{aligned}
$$

with the other terms defined as in lines (A12)-(A15)

$$
\begin{aligned}
T_{2}= & 2 \int \frac{d^{d} q}{(2 \pi)^{d}} \partial_{t} R_{t}(q) G(q)^{2} \Gamma_{q, p,-p-q}^{(3)} \\
& \times\left. G(p+q) \frac{1}{2} \frac{d^{2}}{d p^{2}} \Gamma_{-q,-p, p+q}^{(3)}\right|_{p=0}
\end{aligned}
$$

and

$$
\begin{aligned}
T_{3}= & 2 \int \frac{d^{d} q}{(2 \pi)^{d}} \partial_{t} R_{t}(q) G(q)^{2} \Gamma_{q, p,-p-q}^{(3)} \frac{d}{d p} \\
& \times\left. G(p+q) \frac{d}{d p} \Gamma_{-q,-p, p+q}^{(3)}\right|_{p=0}
\end{aligned}
$$

and

$T_{4}=\left.\int \frac{d^{d} q}{(2 \pi)^{d}} \partial_{t} R_{t}(q) G(q)^{2} G(p+q)\left(\frac{d}{d p} \Gamma_{-q,-p, p+q}^{(3)}\right)^{2}\right|_{p=0}$

and

$$
T_{5}=-\frac{1}{2} \int \frac{d^{d} q}{(2 \pi)^{d}} G(q) \frac{1}{2} \frac{d^{2}}{d p^{2}} \Gamma_{q, p,-p,-q}^{(4)} G(q) \partial_{t} R_{k}(q) .
$$

The most complex term to evaluate is $T_{1}$ we will then left it aside for the moment. It is then convenient to pursue the computation in reverse order. After deriving the vertex term in (A20), we obtain

$$
T_{5}=-\frac{Z^{(2)}}{2} \int \frac{d^{d} q}{(2 \pi)^{d}} G(q)^{2} \partial_{t} R_{k}(q) .
$$

we should then pass to spherical coordinates, integrate over the angular variables and finally transform the integration variable accordingly to $q^{2}=x$,

$$
T_{5}=-Z^{(2)} \frac{s_{d}}{4} \int x^{\frac{d}{2}-1} G(x)^{2} \partial_{t} R_{k}(x) d x=-Z^{(2)} \frac{s_{d}}{4} Q_{\frac{d}{2}}\left[G^{2} \dot{R}\right] .
$$

where we introduced the Mellin transformation, 


$$
Q_{m}[f]=\frac{1}{\Gamma(m)} \int x^{m-1} f(x) d x
$$

and the short hand notation $\dot{A}=\partial_{t} A$. Applying the same notation to (A19) we obtain,

$$
\begin{aligned}
T_{4} & =\left(Z^{(1)}\right)^{2} \frac{s_{d}}{2 d} \int x^{\frac{d}{2}} \partial_{t} R_{t}(x) G(x)^{3} d x \\
& =\left(Z^{(1)}\right)^{2} \frac{s_{d}}{2 d} Q_{\frac{d}{2}+1}\left[G^{3} \dot{R}\right]
\end{aligned}
$$

while for (A18)

$$
\begin{aligned}
T_{3}= & \left(Z^{(1)}\right)^{2} \frac{2 s_{d}}{d} \int x^{\frac{d}{2}+1} \partial_{t} R_{t}(x) G(x)^{2} G_{x}(x) d x \\
& +Z^{(1)} V^{(3)} \frac{2 s_{d}}{d} \int x^{\frac{d}{2}} \partial_{t} R_{t}(x) G(x)^{2} G_{x}(x) d x \\
= & \left(Z^{(1)}\right)^{2} \frac{2 s_{d}}{d} Q_{\frac{d}{2}+2}\left[G^{2} G_{x} \dot{R}\right]+Z^{(1)} V^{(3)} \frac{2 s_{d}}{d} \\
& \times Q_{\frac{d}{2}+1}\left[G^{2} G_{x} \dot{R}\right]
\end{aligned}
$$

and for (A17)

$$
\begin{aligned}
T_{2}= & \left(Z^{(1)}\right)^{2} s_{d} \int x^{\frac{d}{2}} \partial_{t} R_{t}(x) G(x)^{3} d x+Z^{(1)} V^{(3)} s_{d} \\
& \times \int x^{\frac{d}{2}-1} \partial_{t} R_{t}(x) G(x)^{3} d x \\
= & \left(Z^{(1)}\right)^{2} s_{d} Q_{\frac{d}{2}+1}\left[G^{3} \dot{R}\right]+Z^{(1)} V^{(3)} s_{d} Q_{\frac{d}{2}}\left[G^{3} \dot{R}\right]
\end{aligned}
$$

The computation of $T_{1}$ needs some more efforts, we should firstly write the Green function form explicitly

$$
G(p+q)=\frac{1}{Z(\varphi)|p+q|^{2}+U_{k}^{(2)}(\varphi)+R_{t}(p+q)},
$$

We can transform the derivative using the definitions

$$
x=\left(p^{2}+q^{2}+2 p q \cos \theta\right),
$$

We use the equivalence

$$
\frac{1}{2} \frac{d^{2}}{d p^{2}}=\frac{1}{2}\left(\frac{d^{2} x}{d p^{2}} \frac{d}{d x}+\left(\frac{d x}{d p}\right)^{2} \frac{d^{2}}{d x^{2}}\right)
$$

The derivatives are

$$
\begin{aligned}
& \left.\frac{d x}{d p}\right|_{0}=2 q \cos \theta \\
& \left.\frac{d^{2} x}{d p^{2}}\right|_{0}=2 .
\end{aligned}
$$

We can also explicitly compute the derivatives of the propagator with respect to $x$,

$$
\begin{aligned}
\frac{d}{d x} G(q+p)= & G_{x}=-G(x)^{2}\left(Z(\varphi)+R_{t, x}(x)\right), \\
\frac{d^{2}}{d x^{2}} G(q+p)= & G_{x x}=2 G(x)^{3}\left(Z(\varphi)+R_{t, x}(x)\right)^{2} \\
& -G(x)^{2} R_{t, x x}(x)
\end{aligned}
$$

The same must be done for the vertexes,

$$
\begin{aligned}
& \left.\frac{1}{2} \frac{d^{2}}{d p^{2}} \Gamma_{-q,-p, p+q}^{(3)}\right|_{p=0}=Z^{(1)}(\varphi), \\
& \left.\frac{1}{2} \frac{d^{2}}{d p^{2}} \Gamma_{-q,-p, p+q}^{(4)}\right|_{p=0}=Z^{(2)}(\varphi) .
\end{aligned}
$$

We can then rewrite the $T_{1}$ coefficient in the following way,

$$
\begin{aligned}
T_{1}= & \int \frac{d^{d} q}{(2 \pi)^{d}} \partial_{t} R_{t}(q) G(q)^{2}\left(Z^{(1)} x+V^{(3)}\right)^{2} \\
& \times\left.\left(G_{x}+2 q^{2} \cos ^{2} \theta G_{x x}\right)\right|_{p=0} .
\end{aligned}
$$

When evaluated in $p=0$ we obtain $x=q^{2}$, we then use latter relation and we rewrite the integrals using only the $x$ variable, we also integrate over the angular variables obtaining

$$
\begin{aligned}
T_{1}= & \frac{s_{d}}{2} \int x^{\frac{d}{2}-1} d x \partial_{t} R_{t}(x) G(x)^{2}\left(Z^{(1)} x+V^{(3)}\right)^{2}\left(G_{x}(x)+\frac{2}{d} x G_{x x}(x)\right) \\
= & \left(Z^{(1)}\right)^{2} \frac{s_{d}}{2} Q_{\frac{d}{2}+2}\left[G^{2} G_{x} \dot{R}\right]+\left(Z^{(1)}\right)^{2} \frac{s_{d}}{d} Q_{\frac{d}{2}+3}\left[G^{2} G_{x x} \dot{R}\right]+Z^{(1)} V^{(3)} s_{d} Q_{\frac{d}{2}+1}\left[G^{2} G_{x} \dot{R}\right] \\
& +Z^{(1)} V^{(3)} \frac{2 s_{d}}{d} Q_{\frac{d}{2}+2}\left[G^{2} G_{x x} \dot{R}\right]+\left(V^{(3)}\right)^{2} \frac{s_{d}}{2} Q_{\frac{d}{2}}\left[G^{2} G_{x} \dot{R}\right]+\left(V^{(3)}\right)^{2} \frac{s_{d}}{d} Q_{\frac{d}{2}+1}\left[G^{2} G_{x x} \dot{R}\right] .
\end{aligned}
$$

We finally obtain for the flow equations,

$$
\partial_{t} V_{k}(\varphi)=\frac{1}{2} \frac{Q_{\frac{d}{2}}[G \dot{R}]}{(4 \pi)^{d / 2}}
$$




$$
\begin{aligned}
\partial_{t} Z_{k}(\varphi)= & \frac{Z^{(1)}(\varphi)^{2}}{(4 \pi)^{d / 2}}\left(\frac{1}{2} Q_{\frac{d}{2}+1}\left[G^{2} \dot{R}\right]+(d+2) Q_{\frac{d}{2}+2}\left[G^{2} G_{x} \dot{R}\right]+d Q_{\frac{d}{2}+1}\left[G^{3} \dot{R}\right]\right. \\
& \left.+\frac{d}{2}\left(\frac{d}{2}+1\right) Q_{\frac{d}{2}+2}\left[G^{2} G_{x} \dot{R}\right]+\left(\frac{d}{2}+2\right)\left(\frac{d}{2}+1\right) Q_{\frac{d}{2}+3}\left[G^{2} G_{x x} \dot{R}\right]\right)-\frac{Z^{(2)}(\varphi)}{2(4 \pi)^{d / 2}} Q_{\frac{d}{2}}\left[G^{2} \dot{R}\right] \\
& +\frac{Z^{(1)}(\varphi) V^{(3)}(\varphi)}{(4 \pi)^{d / 2}}\left(2 Q_{\frac{d}{2}+1}\left[G^{2} G_{x} \dot{R}\right]+\frac{2}{d} Q_{\frac{d}{2}}\left[G^{3} \dot{R}\right]+d Q_{\frac{d}{2}+1}\left[G^{2} G_{x} \dot{R}\right]+(d+2) Q_{\frac{d}{2}+2}\left[G^{2} G_{x x} \dot{R}\right]\right) \\
& +\frac{V^{(3)}(\varphi)^{2}}{(4 \pi)^{d / 2}}\left(Q_{\frac{d}{2}}\left[G^{2} G_{x} \dot{R}\right]+Q_{\frac{d}{2}+1}\left[G^{2} G_{x x} \dot{R}\right]\right) .
\end{aligned}
$$

The derivatives of the propagator are

$$
\begin{aligned}
\frac{d}{d x} G(q+p)= & G_{x}=-G(x)^{2}\left(Z(\varphi)+R_{t, x}(x)\right), \\
\frac{d^{2}}{d x^{2}} G(q+p)= & G_{x x}=2 G(x)^{3}\left(Z(\varphi)+R_{t, x}(x)\right)^{2} \\
& -G(x)^{2} R_{t, x x}(x) .
\end{aligned}
$$

To further proceed it is necessary to specify the cutoff functions. The regulator employed in our calculations is the linear or optimized cutoff

$$
\begin{aligned}
R_{k}(x) & =Z_{k}\left(k^{2}-x\right) \theta\left(k^{2}-x\right) \\
R_{k, x}(x) & =-Z_{k} \theta\left(k^{2}-x\right) \\
R_{k, x x}(x) & =Z_{k} \delta\left(k^{2}-x\right) \\
\dot{R}_{k}(x) & =2 Z_{k} k^{2} \theta\left(k^{2}-x\right)-\dot{Z}_{k}\left(k^{2}-x\right) \theta\left(k^{2}-x\right),
\end{aligned}
$$

where the terms proportional to $\left(k^{2}-x\right) \delta\left(k^{2}-x\right)$ and their successive derivatives have been neglected since they are zero in the distribution sense. The same result can be formally obtained regularizing the $\delta$-function via its heat kernel representation and taking the singular limit at the end of the calculation.

In order to give the expressions for the flow equations, one should consider the explicit form of the $\mathrm{Q}$ functionals in the optimized cutoff case

$$
\begin{aligned}
Q_{m} & {\left[G^{n} \dot{R}\right] } \\
& =\int_{0}^{k^{2}} x^{m-1} d x \frac{2 Z_{k} k^{2}+\dot{Z}_{k}\left(k^{2}-x\right)}{\left(\left(Z_{k}(\varphi)-Z_{k}\right) x+Z_{k} k^{2}+V_{k}^{(2)}(\varphi)\right)^{n}},
\end{aligned}
$$

$$
\begin{aligned}
& Q_{m}\left[G^{n} G_{x} \dot{R}\right] \\
& =-\int_{0}^{k^{2}} x^{m-1} d x \frac{\left(2 Z_{k} k^{2}+\dot{Z}_{k}\left(k^{2}-x\right)\right)\left(Z_{k}(\varphi)-Z_{k}\right)}{\left(\left(Z_{k}(\varphi)-Z_{k}\right) x+Z_{k} k^{2}+V_{k}^{(2)}(\varphi)\right)^{n+2}}, \\
& Q_{m}\left[G^{n} G_{x x} \dot{R}\right] \\
& =\int_{0}^{k^{2}} x^{m-1} d x \frac{2\left(2 Z_{k} k^{2}+\dot{Z}_{k}\left(k^{2}-x\right)\right)\left(Z_{k}(\varphi)-Z_{k}\right)^{2}}{\left(\left(Z_{k}(\varphi)-Z_{k}\right) x+Z_{k} k^{2}+V_{k}^{(2)}(\varphi)\right)^{n+3}} \\
& \quad-\frac{2 Z_{k}^{2} \delta\left(k^{2}-x\right)}{\left(\left(Z_{k}(\varphi)-Z_{k}\right) x+Z_{k} k^{2}+V_{k}^{(2)}(\varphi)\right)^{n+2}} .
\end{aligned}
$$

The only ill-defined expression in the latter equations is on line (A50), where the $\delta$ function is evaluated at the boundary of the integration measure. In our convention, this expression has to be evaluated as

$$
\int_{0}^{k^{2}} f(x) \delta\left(x-k^{2}\right)=\frac{1}{2} f\left(k^{2}\right) .
$$

Latter definition is consistent with the heat kernel representation of the $\delta$ function as well as with other evaluation schemes appeared in literature [41]. The integrated expressions for the $\mathrm{Q}$ functionals are given in the next section.

\section{APPENDIX B: Q FUNCTIONALS}

The $\mathrm{Q}$ functionals appearing in the beta functionals $\beta_{V}$ and $\beta_{Z}$ of Sec. II can be evaluated analytically when the linear, or Litim, cutoff

$$
R_{k}(x)=Z_{k}\left(k^{2}-x\right) \theta\left(k^{2}-x\right)
$$

is employed. The explicit expressions are 


$$
\begin{aligned}
Q_{n}\left[G_{k}^{m} \partial_{t} R_{k}\right] & =\frac{k^{2(n-m+1)} Z_{k}^{1-m}}{\Gamma(n)}\left\{(2-\eta) q_{n, m}(\zeta, \omega)+\eta q_{n+1, m}(\zeta, \omega)\right\} \\
Q_{n}\left[G_{k}^{m} G_{k}^{\prime} \partial_{t} R_{k}\right] & =-\frac{k^{2(n-m-1)} Z_{k}^{-m}}{\Gamma(n)}(\zeta-1)\left\{(2-\eta) q_{n, m+2}(\zeta, \omega)+\eta q_{n+1, m+2}(\zeta, \omega)\right\} \\
Q_{n}\left[G_{k}^{m} G_{k}^{\prime \prime} \partial_{t} R_{k}\right] & =2 \frac{k^{2(n-m-2)} Z_{k}^{-m}}{\Gamma(n)}(\zeta-1)^{2}\left\{(2-\eta) q_{n, m+3}(\zeta, \omega)+\eta q_{n+1, m+3}(\zeta, \omega)\right\}-\frac{k^{2(n-m-2)} Z_{k}^{-m}}{\Gamma(n)} \frac{1}{(\zeta+\omega)^{m+2}},
\end{aligned}
$$

where we defined the threshold integral

$$
q_{n, m}(\zeta, \omega) \equiv \frac{1}{n(1+\omega)^{m}}{ }_{2} F_{1}\left(m, n ; n+1 ; \frac{1-\zeta}{1+\omega}\right),
$$

with $\omega=\frac{V_{k}^{\prime \prime}(\phi)}{k^{2} Z_{k}}$ and $\zeta=\frac{Z_{k}(\phi)}{Z_{k}}$. In $d=2,3$, one obtains the expressions given in the following section.

\section{APPENDIX C: BETA FUNCTIONALS}

Here, we report the explicit linear cutoff expressions, in two and three dimensions, for the dimensionless beta functionals $\beta_{v}$ and $\beta_{\zeta}$ defined in Sec. II.

\section{1. $d=2$}

$$
\begin{aligned}
\beta_{v}= & -2 v+\frac{\eta}{2} \varphi v^{\prime}+\frac{1}{8 \pi}\left\{\frac{\eta}{\zeta-1}-\frac{2+\eta \omega-(2-\eta) \zeta}{(\zeta-1)^{2}} \log \frac{\zeta+\omega}{1+\omega}\right\} \\
\beta_{\zeta}= & \eta \zeta+\frac{\eta}{2} \varphi \zeta^{\prime}+\frac{\zeta^{\prime \prime}}{8 \pi}\left\{\frac{2+\eta \omega-(2-\eta) \zeta}{(\zeta-1)(1+\omega)(\zeta+\omega)}-\frac{\eta}{(\zeta-1)^{2}} \log \frac{\zeta+\omega}{1+\omega}\right\}+\frac{\left(v^{\prime \prime \prime}\right)^{2}}{8 \pi}\left\{\frac{2-\eta}{3(\zeta+\omega)^{3}}-\frac{2-\eta}{3(1+\omega)^{3}}-\frac{2 \zeta}{(\zeta+\omega)^{4}}\right\} \\
& +\frac{v^{\prime \prime \prime} \zeta^{\prime}}{4 \pi}\left\{\frac{4-\eta}{(1+\omega)^{2}(\zeta+\omega)^{4}} \omega^{3}+\frac{\zeta(18-7 \eta)-2 \eta+12}{3(1+\omega)^{2}(\zeta+\omega)^{4}} \omega^{2}+\frac{2 \zeta^{2}(8-3 \eta)+2 \zeta(2-\eta)+4-\eta}{3(1+\omega)^{2}(\zeta+\omega)^{4}} \omega\right. \\
& \left.+\frac{2 \zeta^{3}(2-\eta)+4 \zeta^{2}-\zeta(2+\eta)}{3(1+\omega)^{2}(\zeta+\omega)^{4}}\right\}+\frac{\left(\zeta^{\prime}\right)^{2}}{8 \pi}\left\{-\frac{3 \eta}{(\zeta-1)^{2}(1+\omega)(\zeta+\omega)^{4}} \omega^{4}-\frac{3 \eta(7 \zeta+1)}{2(\zeta-1)^{2}(1+\omega)(\zeta+\omega)^{4}} \omega^{3}\right. \\
& +\frac{\zeta^{2}(30-83 \eta)-\zeta(23 \eta+60)-2 \eta+30}{6(\zeta-1)^{2}(1+\omega)(\zeta+\omega)^{4}} \omega^{2}+\frac{\zeta^{3}(36-47 \eta)-\zeta^{2}(25 \eta+60)+2 \zeta(6+\eta)+12-2 \eta}{6(\zeta-1)^{2}(1+\omega)(\zeta+\omega)^{4}} \omega \\
& \left.+\frac{9 \zeta^{4}(2-\eta)-\zeta^{3}(11 \eta+36)+\zeta^{2}(18+4 \eta)-2 \zeta \eta}{6(\zeta-1)^{2}(1+\omega)(\zeta+\omega)^{4}}+\frac{3 \eta}{(\zeta-1)^{3}} \log \frac{\zeta+\omega}{1+\omega}\right\} .
\end{aligned}
$$

\section{2. $d=3$}

$$
\beta_{v}=-3 v+\frac{1+\eta}{2} \varphi v^{\prime}+\frac{1}{4 \pi^{2}}\left\{-\frac{6+(1+3 \omega) \eta-2(3-\eta) \zeta}{3(\zeta-1)^{2}}+\frac{2+\eta \omega-(2-\eta) \zeta}{(1-\zeta)^{5 / 2}} \sqrt{1+\omega} \operatorname{arctanh} \sqrt{\frac{1-\zeta}{1+\omega}}\right\}
$$




$$
\begin{aligned}
& \beta_{\zeta}=\eta \zeta+\frac{1+\eta}{2} \varphi \zeta^{\prime}+\frac{\zeta^{\prime \prime}}{8 \pi^{2}}\left\{\frac{\zeta(2-3 \eta)-2-3 \eta \omega}{(\zeta-1)^{2}(\zeta+\omega)}+\frac{2+\eta(2+3 \omega)-\zeta(2-\eta)}{(1-\zeta)^{5 / 2} \sqrt{\omega+1}} \operatorname{arctanh} \sqrt{\frac{1-\zeta}{1+\omega}}\right\} \\
& +\frac{\left(v^{\prime \prime \prime}\right)^{2}}{(4 \pi)^{2}}\left\{\frac{-3 \zeta(\eta-2)+\eta(\omega+4)-6}{24(1-\zeta)^{3 / 2}(\omega+1)^{5 / 2}} \operatorname{arctanh} \sqrt{\frac{1-\zeta}{1+\omega}}-\frac{1}{12} \frac{\eta-1}{(\zeta-1)^{2}(\omega+1)}+\frac{1}{12} \frac{\eta-1}{(\zeta-1)^{2}(\zeta+\omega)}\right. \\
& \left.+\frac{1}{18} \frac{\eta-2}{(\zeta-1)(\omega+1)^{2}}-\frac{2}{9} \frac{(\eta-3)}{(\zeta+\omega)^{3}}-\frac{4}{3} \frac{\zeta}{(\zeta+\omega)^{4}}+\frac{1}{12} \frac{1}{(\zeta-1)(\zeta+\omega)^{2}}\right\} \\
& +\frac{v^{\prime \prime \prime} \zeta^{\prime}}{8 \pi^{2}}\left\{-\frac{7}{12} \frac{\zeta(\eta-2)-\eta(3 \omega+4)+2}{(1-\zeta)^{5 / 2}(\omega+1)^{3 / 2}} \operatorname{arctanh} \sqrt{\frac{1-\zeta}{1+\omega}}-\frac{7}{12} \frac{\eta-2}{(\zeta-1)^{2}(\omega+1)}-\frac{4}{9} \frac{\eta-5}{(\zeta+\omega)^{3}}-\frac{8}{3} \frac{\zeta}{(\zeta+\omega)^{4}}\right. \\
& \left.-\frac{7}{6} \frac{\eta+1}{(\zeta-1)^{2}(\zeta+\omega)}-\frac{7}{3} \frac{1}{(\zeta-1)(\zeta+\omega)^{2}}\right\}+\frac{\left(\zeta^{\prime}\right)^{2}}{8 \pi^{2}}\left\{\frac{49}{24} \frac{\zeta(\eta-2)+\eta(5 \omega+4)+2}{(1-\zeta)^{7 / 2} \sqrt{\omega+1}} \operatorname{arctanh} \sqrt{\frac{1-\zeta}{1+\omega}}\right. \\
& \left.-\frac{2}{9} \frac{\eta-7}{(\zeta+\omega)^{3}}+\frac{245 \eta}{24(\zeta-1)^{3}}-\frac{4}{3} \frac{\zeta}{(\zeta+\omega)^{4}}-\frac{49}{32} \frac{\eta+3}{(\zeta-1)^{2}(\zeta+\omega)}-\frac{49}{18} \frac{1}{(\zeta-1)(\zeta+\omega)^{2}}\right\} \text {. }
\end{aligned}
$$

[1] K. G. Wilson, Rev. Mod. Phys. 47, 773 (1975); 55, 583 (1983).

[2] N. Tetradis and C. Wetterich, Nucl. Phys. B422, 541 (1994).

[3] T. R. Morris, Phys. Lett. B 329, 241 (1994).

[4] J. Berges, N. Tetradis, and C. Wetterich, Phys. Rep. 363, 223 (2002).

[5] A. Schwenk and J. Polonyi, Lect. Notes Phys. 852 (2012).

[6] P. Kopietz, L. Bartosch, and F. Schtz, Lect. Notes Phys. 798 (2010).

[7] T. R. Morris, Phys. Rev. Lett. 77, 1658 (1996).

[8] A. Codello and G. D’Odorico, Phys. Rev. Lett. 110, 141601 (2013).

[9] A. Codello, N. Defenu, and G. D’Odorico, Phys. Rev. D 91, 105003 (2015).

[10] A. Codello, J. Phys. A 45, 465006 (2012).

[11] T. R. Morris, Phys. Lett. B 345, 139 (1995).

[12] R. Neves, Y. Kubyshin, and R. Potting, arXiv:hep-th/ 9811151.

[13] A. Bonanno and D. Zappala, Phys. Lett. B 504, 181 (2001).

[14] C. Bervillier, B. Boisseau, and H. Giacomini, Nucl. Phys. B801, 296 (2008).

[15] S. Yabunaka and B. Delamotte, Phys. Rev. Lett. 119, 191602 (2017).

[16] A. Katsis and N. Tetradis, Phys. Lett. B 780, 491 (2018).

[17] L. Canet, B. Delamotte, D. Mouhanna, and J. Vidal, Phys. Rev. D 67, 065004 (2003).

[18] D. F. Litim and D. Zappala, Phys. Rev. D 83, 085009 (2011).

[19] D. F. Litim, Phys. Lett. B 486, 92 (2000); Phys. Rev. D 64, 105007 (2001).

[20] N. Defenu et al. J. High Energy Phys. 15 (2015) 141.

[21] T. Hellwig, A. Wipf, and O. Zanusso, Phys. Rev. D 92, 085027 (2015).

[22] T. R. Morris, Nucl. Phys. B, Proc. Suppl. 42, 811 (1995).
[23] T. R. Morris, Nucl. Phys. B495, 477 (1997).

[24] G. Mussardo, Statistical Field Theory: An Introduction to Exactly Solved Models in Statistical Physics (Oxford University Press, New York, 2010).

[25] W. H. Press et al., Numerical Recipes. The Art of Scientific Computing (Cambridge University Press, Cambridge, England, 2007), 3nd ed.

[26] S. Nagy, J. Krizsan, and K. Sailer, J. High Energy Phys. 07 (2012) 102.

[27] A. Pelissetto and E. Vicari, Phys. Rep. 368, 549 (2002).

[28] S. El-Showk, M. F. Paulos, D. Poland, S. Rychkov, D. Simmons-Duffin, and A. Vichi, J. Stat. Phys. 157, 869 (2014).

[29] J. O'Dwyer and H. Osborn, Ann. Phys. (Amsterdam) 323, 1859 (2008).

[30] A. Codello, M. Safari, G. P. Vacca, and O. Zanusso, J. High Energy Phys. 04 (2017) 127.

[31] A. Codello, M. Safari, G. P. Vacca, and O. Zanusso, Eur. Phys. J. C 78, 30 (2018).

[32] A. Codello, M. Safari, G. P. Vacca, and O. Zanusso, Phys. Rev. D 96, 081701 (2017).

[33] L. Zambelli, arXiv:1510.09151.

[34] L. Zambelli and O. Zanusso, Phys. Rev. D 95, 085001 (2017).

[35] N. Defenu, A. Trombettoni, and A. Codello, Phys. Rev. E 92, 052113 (2015).

[36] N. Defenu, A. Trombettoni, and S. Ruffo, Phys. Rev. B 94, 224411 (2016).

[37] N. Defenu, A. Trombettoni, and S. Ruffo, Phys. Rev. B 96, 104432 (2017).

[38] R. B. A. Zinati and A. Codello, J. Stat. Mech. 1801, 013206 (2018).

[39] L. Fei, S. Giombi, and I. R. Klebanov, Phys. Rev. D 90, 025018 (2014).

[40] R. Percacci and G. P. Vacca, Phys. Rev. D 90, 107702 (2014).

[41] N. Christiansen, arXiv:1612.06223. 